\title{
Salidroside induces apoptosis in human gastric cancer cells via the downregulation of ENO1/PKM2/GLUT1 expression
}

Ziying Dai ${ }^{a}$, Xuan Zhang ${ }^{b, f}$, Wuyan $\mathrm{Li}^{c}$, Junxia Tang ${ }^{a}$, Tingting $\operatorname{Pan}^{a}$, Chenru Ma ${ }^{a}$, Quanlin Guan ${ }^{d, e^{*}}$

a. The First Clinical Medical Collage of Lanzhou University, Lanzhou, Gansu 730000, P.R. China

b. School of life science, Lanzhou University, Lanzhou, Gansu 730000, P.R. China c. Center for Inflammation, Translational and Clinical Lung Research, Temple University School of Medicine, Philadelphia, PA19140, USA

d. The First Hospital of Lanzhou University, Lanzhou, Gansu 730000, P.R. China

e. Key Laboratory for Gastrointestinal Diseases of Gansu Province, Lanzhou University, Lanzhou, Gansu 730000, P.R. China

f. Research and Experimental Centre Gansu University of Chinese Medicine, Lanzhou, Gansu 730000, P.R. China

Correspondence to: Dr Quanlin Guan, Department of Surgical Oncology, The First Hospital of Lanzhou University, NO.1 Donggang West Road, Lanzhou 730000, Gansu Province, P.R. China.

E-mail: guanquanlin@ 163.com 


\section{Abstract}

Salidroside is reported to have a wide range of pharmacological properties and has been proven to play a key anti-cancer effect. This study investigated the effects of purified salidroside, an ingredient of Rhodiola rosea, on the proliferation of two human gastric cancer cell lines and further investigating its possible molecular mechanisms. We verified that salidroside exerts a dose-dependent inhibitory effect on the proliferation of SGC-7901 and MKN-45 human gastric cancer cells. Moreover, salidroside can induce cell apoptosis, which was accompanied by an increase in nuclear fragmentation. In addition, salidroside inhibited glycolysis, as evidenced by the reduced expression levels of the glycolysis-related enzymes pyruvate kinase isoenzyme M2 (PKM2), enolase 1 (ENO1) and glucose transporter 1 (GLUT1), which could play important roles in the metabolism of gastric cancer cells. Further investigation showed that salidroside exerted potent anti-proliferative effects by inhibiting glycolysis in human gastric cancer cells in vitro. In vivo, xenograft tumors treated with salidroside were significantly smaller than those in the control animals. Therefore, salidroside could be a promising therapeutic prospect in the treatment of gastric cancer.

Key words: salidroside; gastric cancer; glycolysis; ENO1; PKM2; GLUT1 


\section{Introduction}

Gastric cancer is a common malignancy especially in China, which has a high mortality rate worldwide ${ }^{1)}$. Some studies have suggested that cancer is a metabolic disease. The dysregulation of intracellular metabolism, including abnormal glucose metabolism, eventually results in the occurrence of cancer ${ }^{2)}$. Cancer cells have various metabolic requirements different from those of healthy cells to satisfy the higher energy demands aimed at proliferation ${ }^{3)}$.Different from normal cells, Even when oxygen is abundant, most cancer cells, produce energy via the extremely inefficient pathway of glycolysis ${ }^{4,5)}$. As glycolysis is a common means for cancer cells to obtain energy, an increasing number of studies are currently exploring glycolysis-related genes such as enolase (ENO), pyruvate kinase (PKM) and glucose transporter (GLUTs) as therapeutic targets to inhibit the occurrence and development of gastric cancer ${ }^{6-9)}$.

Rhodiola rosea is widely distributed at high elevations. Salidroside (p-hydroxy phenylethyl- $\beta$-D-glucoside) is one of the most effective components in the isolation of R. rosea. ${ }^{10)}$. Numerous studies have contended that the benefits of salidroside include a high medicinal value and anti-radiation, anti-virus, antitumour, protective effects against neuronal death and immune-regulating activity ${ }^{11-13)}$. Numerous anticancer studies have observed the effects of salidroside on the cancer cell cycle and proved that salidroside even induces apoptosis and autophagy in human gastric cancer cells.

In our study, the experiment investigated the effects of salidroside on the proliferation, migration and invasion of SGC-7901 and MKN-45 cells, along with the 
potential molecular mechanisms. Salidroside inhibited SGC-7901 and MKN-45 cell proliferation, migration and invasion in a dose-dependent manner through the suppression of ENO1, PKM2 and GLUT1 expression as well as the inhibition of glycolysis signalling pathways. This study provides a new perspective for the application of salidroside in tumour treatment.

\section{Materials and methods}

\subsection{Cell culture and treatment}

Gastric cell lines SGC-7901 and MKN-45 were purchased from Shanghai Cell Bank, Chinese Academy of Sciences and used for this study. Cells were cultured for $24 \mathrm{~h}$ in an incubator with $5 \% \mathrm{CO} 2,37{ }^{\circ} \mathrm{C}$ and $95 \%$ humidity for subsequent experiments. The cells were cultured in the medium supplemented with $10 \%$ foetal bovine serum DMEM (HyClone, Utah, USA) and cultured in an incubator with 5\% $\mathrm{CO}^{2}, 37^{\circ} \mathrm{C}$ and $95 \%$ humidity for $24 \mathrm{~h}$. Salidroside (purity $>99 \%$ ) was obtained from Shanghai Yuanye Biological Co., Ltd. Salidroside was prepared different concentrations in PBS intended for subsequent experiments.

\subsection{Cell viability assay.}

SGC-7901 and MKN-45 cells were cultured in 96-well plates at a density of $3 \times 10^{3}$. Then dividing into control group and different salidroside treatment group (10 $\mu \mathrm{M}, 20 \mu \mathrm{M}, 40 \mu \mathrm{M}, 80 \mu \mathrm{M})$ treated with different concentrations of salidroside for 24

h. The growth inhibitory effect of salidroside on cancer cells was measured by a Cell Counting Kit-8 (CCK-8) assay (Dojindo, Kumamoto, Japan). The cell viability was calculated according to the following formula: Cell viability $(\%)=(\mathrm{D}$ experimental group 
$\left.-\mathrm{D}_{\text {blank control }}\right) /\left(\mathrm{D}_{\text {control group }}-\mathrm{D}_{\text {blank control }}\right) \times 100 \%$

\subsection{Wound healing assay.}

SGC-7901 and MKN-45 cells were inoculated in 6-well plates and cultured for $24 \mathrm{~h}$ until the cells were full confluence. The cell monolayer was scraped in a straight line with a p200 pipet tip to create a wound. After that, Damaged cells were removed by washing with PBS (phosphate-buffered saline), and remaining cells in the plate were treated by different concentrations of salidroside $(0,10,20,40,80 \mu \mathrm{M})$ with DMEM serum-free for 24 h.Image of migration across the injure areas were taken by the inverted microscope(Olympus Corporation, Japan $)^{14)}$. Calculate the cell migration rate: $\mathrm{n}$ hour cell migration rate $=(0 \mathrm{~h}$ scratch area $-24 \mathrm{~h}$ scratch area $) / 0 \mathrm{~h}$ scratch area $100 \%$.

\subsection{Cancer cell apoptosis assay in vitro.}

SGC-7901 and MKN-45 cells were seeded in a 12-well cell culture plate and cultured at $37^{\circ} \mathrm{C}$. Cells were then treated with salidroside $(80 \mu \mathrm{M})$ for $24 \mathrm{~h}$. Cells were fixed in $4 \%$ of pre-cooled paraformaldehyde (PFA, Sigma, USA) for $15 \mathrm{~min}$ and washed with PBS. After washing, cells were treated with $0.1 \%$ Triton X-100 for 15 min and were then treated with 5\% BSA blocking solution for $1 \mathrm{~h}$ at room temperature. Then, cells were labelled with a TUNEL kit (Transgene FA201-02, China). after which Hoechst 33258 (Beijing Solarbio Science \& Technology, Beijing, China) at a 1:200 dilution was used to detect cancer cell. Apoptotic cells were labelled green, and the labelled cells were observed using a fluorescence microscope system (Olympus Corporation, Japan). 


\section{5. pH-Xtra glycolysis assay.}

The experiment used pH-Xtra Glycolysis Assay (Agilent Technologies, USA) and mainly analysed the extracellular acidification rate (ECAR). The slope of the experimental time and TR-F values reflect the degree of tumour extracellular acidification and indirectly represent the glycolytic ability of the tumour. SGC-7901 and MKN-45 cells were seeded in 96-well cell culture plates at a density of $6 \times 10^{4}$ cells per well and added $200 \mu \mathrm{l}$ medium at $37^{\circ} \mathrm{C} \mathrm{CO}_{2}$ incubator for the night. Because the residual $\mathrm{CO}_{2}$ could contribute to acidification. Thus, before glycolysis determined, the cells should be in $37^{\circ} \mathrm{C}, 95 \%$ humidity without carbon dioxide for 2 hours, so as to eliminate the residual $\mathrm{CO}_{2}$. Then Cells were washed twice using $100 \mu$ of respiration buffer per well for each wash. After removing the second wash, adding $90 \mu \mathrm{l}$ of fresh respiration buffer. $10 \mu \mathrm{l}$ of reconstituted $\mathrm{pH}-\mathrm{Xtra}$ reagent was added to all wells except the wells used as the blank controls. Then, $10 \mu$ of respiration buffer was added to the blank control wells. $80 \mu \mathrm{M}$ salidroside (typically 1-10 $\mu \mathrm{l}$ ) was added at this point. The plate was then immediately read in a fluorescence plate reader. The plate was measured kinetically for $>120 \mathrm{~min}$. When the measurement was completed, the plate was removed, and the measured data were saved to a file.

\subsection{Cell immunofluorescence assay.}

SGC-7901 and MKN-45 cells fixed in 4\% pre-cooled PFA for 15 min and rinsed with PBS. After washing, cells were treated by $0.25 \%$ Triton $\mathrm{X}-100$ at room temperature for $30 \mathrm{~min}$. then blocked by $5 \%$ BSA for $2 \mathrm{~h}$. Primary antibody incubated cells overnight at $4{ }^{\circ} \mathrm{C}$. The primary antibodies were anti-ENO1 (1:500; GeneTex, 
USA) and anti-PKM2 and anti-GLUT1 (1:400, Abcam, Cambridge, MA, USA). Fluorescence microscopy was used to visualize the immunofluorescence staining.

\subsection{Gene expression analysis.}

Total RNA was extracted by Trizol reagent (Invitrogen, Carlsbad, USA), and the concentration and purity of RNA were determined by NanoDrop2000 microspectrophotometer ( Thermo Fisher, USA ) .The total RNA was reverse transcribed to cDNA using a PrimeScript RT reagent kit (TaKaRa, Tokyo, Japan).

The target gene expression was detected by real-time quantitative PCR.Using a SYBR Premix Ex Taq II Kit (TaKaRa) and LightCycler 480 instrument (Roche, Basel, Switzerland). According to the instructions, the amplification conditions were following: pre-denaturation $95^{\circ} \mathrm{C}$ for $30 \mathrm{~s}$, then denaturation 45 cycles at $95^{\circ} \mathrm{C}$ for $5 \mathrm{~s}$ and $58^{\circ} \mathrm{C}$ for $30 \mathrm{~s}$, followed by $95^{\circ} \mathrm{C}, 1 \mathrm{~min} ; 55^{\circ} \mathrm{C}, 30 \mathrm{~s} ; 95^{\circ} \mathrm{C}, 30 \mathrm{~s}$. The $2^{-\Delta \Delta \mathrm{Ct}}$ method was used to calculate the expression levels of the target genes ${ }^{15)}$. The glyceraldehyde-3-phosphate dehydrogenase (GAPDH) was used as the reference. The primer sequences are shown in Table 1. Each sample was measured in duplicate, and the experiment was conducted in triplicate. The primers used in this study are shown in Table. 1.

\subsection{Western blot analysis.}

Cells were lysed in RIPA buffer containing protease inhibitors for 20 minutes at $4^{\circ} \mathrm{C}$. According to the manufacturer's instructions, the protein concentration was determined using the BCA protein detection kit (Thermo Fisher Scientific, MA, USA). The protein extract was separated by $10 \%$ SDS-PAGE $(40 \mathrm{mg} / \mathrm{l})$ and transferred to a 
polyvinylidene fluoride (PVDF) membrane (Sangong Biotechnology, Shanghai, China). The membrane was blocked with PBST containing 5\% skim milk and incubated overnight at $4^{\circ} \mathrm{C}$ with primary antibodies against PKM2, GLUT1, and $\beta$-actin (both 1:800; Abcam, Cambridge, MA, USA) and ENO1 (1:800; American GeneTex). Then use horseradish peroxidase (HRP) conjugated secondary antibody (1:500; Zhongshan Jinqiao Biotechnology Co., Ltd., Beijing, China) The signal was detected with enhanced chemiluminescence detection reagent (Thermo Fisher Scientific, MA, USA) at room temperature for 2 hours. ENO1, PKM2, and GLUT1 levels were normalized to endogenous $\beta$-actin levels. The experimental results were visualized with Amersham Imager 680 from GE Healthcare Life Science, USA.

\subsection{Xenograft model.}

Four-week-old BALB/c Nude male mice were maintained under specific pathogen-free (SPF) conditions in the animal care facility at Gansu University of Traditional Chinese Medicine (Lanzhou, China). All animal procedures used in this study were carried out in accordance with the National Institutes of Health guide for the care and use of Laboratory animals (NIH Publications No. 8023, revised 1978) and the ethical number (2021-179). Mice were randomly divided into 4 groups and MKN-45 and SGC-7901 cells were injected subcutaneously into the left flank of each mouse $\left(5 \times 10^{6}\right.$ cells in $150 \mu$ of DMEM serum-free per injection). Then, each mouse was intraperitoneal injected with either $100 \mu \mathrm{l}$ of salidroside $(50 \mathrm{mg} / \mathrm{kg} / \mathrm{d})$ or $100 \mu \mathrm{l}$ of PBS. tumor volumes were measured by per 5 day and and the growth curve of the tumors was plotted. Xenograft tumors were harvested, weighed, and measured after 
first injected salidroside 7 days. The length (L) and width (W) of the tumors were measured 10 days after cell inoculation. The tumour volumes were calculated by the following formula: $\mathrm{V}=\mathrm{L} \times \mathrm{W} \times \mathrm{W} \times 0.5^{16)}$.

\subsection{Immunohistochemistry staining.}

The tumors were removed from the mice and fixed in 4\% PFA, the related expression of tumors was diagnosed by paraffin section. Slides were immersed in xylene and gradient alcohol for dewaxing, and soaked in $3 \% \mathrm{H}_{2} \mathrm{O}_{2}$ to eliminate peroxidase. $0.01 \mathrm{~mol} / \mathrm{L}$ sodium citrate buffer solution $(\mathrm{pH} \mathrm{6.0)}$ was used to antigen retrieval at $90^{\circ} \mathrm{C}$ and rinsed with PBS. After that the slides blocked by 5\% BSA. Primary antibody incubated slides overnight at $4^{\circ} \mathrm{C}$, including ENO1(Abcam, USA 1:2000), HK2(Abcam, USA 1:300), GLUT1(Immunoway, USA 1:100). Secondary antibody incubated at $37^{\circ} \mathrm{C}$ for 1 hour, and rinsed with PBS.Horseradish peroxidase-labeled streptomycin avidin working solution incubated at $37^{\circ} \mathrm{C}$ for 30 min. Stain separation was used to hematoxylin counterstain from DAB antibody-specific stain. Optical microscope was used to visualize the Immunohistochemistry staining.

\subsection{Statistical analysis.}

The data were statistical analysed by SPSS 24.0 software (IBM, Chicago, IL, US A) and GraphPad Prism 9 Software (GraphPad, San Diego, CA, USA).

Values are expressed as the means \pm standard deviations. A value of $\mathrm{P}<0.05$ was considered statistically significant.

\section{Results}




\subsection{Salidroside inhibits growth and induces apoptosis in gastric cancer cells.}

MKN-45 and SGC-7091 gastric cancer cells were treated with salidroside to investigate the activity of salidroside against the cells. Then, cell viability was evaluated by a CCK-8 assay. This assay revealed dose-dependent inhibiting effects of salidroside on the cells following treatment (Figure. 1B). SGC-7901 cell viability was reduced to $92.36 \% \pm 2.22 \%, 83.17 \% \pm 4.42 \%, 72.05 \% \pm 3.12 \%$ and $66.78 \% \pm 1.72 \%$ following treatment with $10 \mu \mathrm{M}, 20 \mu \mathrm{M}, 40 \mu \mathrm{M}$ and $80 \mu \mathrm{M}$ salidroside, respectively, compared with $100 \%$ in the control group. MKN-45 cell viability was decreased to $85.40 \% \pm 3.75 \%, 72.11 \% \pm 3.65 \%, 65.14 \% \pm 2.10 \%$ and $62.90 \% \pm 1.94 \%$ following treatment with $10 \mu \mathrm{M}, 20 \mu \mathrm{M}, 40 \mu \mathrm{M}$ and $80 \mu \mathrm{M}$ salidroside, respectively, compared with $100 \%$ in the control group. Salidroside treatment markedly decreased SGC-7901 and MKN-45 cell viability in a dose-dependent manner.

\subsection{Salidroside suppresses gastric cancer cell migration.}

A wound healing assay was used to determine whether different salidroside concentrations $(0,10,20,40$, and $80 \mu \mathrm{M})$ inhibited the migration of SGC-7901 and MKN-45 cells (Figure. 2A). The cell-free area after treatment was wider in the treated groups than in the corresponding control groups at $24 \mathrm{~h}$. These results demonstrated that salidroside treatment markedly inhibited SGC-7901 and MKN-45 cell migration in a dose-dependent manner. The migration capability of salidroside $(80 \mu \mathrm{M})$ treated cells was markedly suppressed relative to that of the corresponding control cells. The experimental results are shown (Figure.2B). As $80 \mu \mathrm{M}$ salidroside effectively reduced the viability and inhibited the metastasis of gastric cancer cells, this concentration was 
used in subsequent experiments.

3.3. Salidroside promotes the apoptosis of tumour cells, as evidenced by a TUNEL assay.

A TUNEL Apoptosis Cell Kit and Hoechst 33258 were used to further study the apoptotic effect of $24 \mathrm{~h}$ of treatment with $80 \mu \mathrm{M}$ salidroside in SGC-7901 and MKN-45 cells (Figure.3A). As seen from the figure, almost no TUNEL-positive cells were observed in the corresponding control cells, indicating that almost no apoptosis was occurring at this time point. However, many TUNEL-positive cells were observed after salidroside treatment, and the number of apoptotic cells was significantly increased. These results indicate that salidroside exerted an enhancing effect on apoptosis in SGC-7901 and MKN-45 gastric cancer cells.

\subsection{Salidroside inhibits the glycolytic ability of gastric cancer cells.}

To confirm the inhibitory effect of salidroside on glycolysis, cells were treated with $80 \mu \mathrm{M}$ salidroside. The $\mathrm{pH}-\mathrm{Xtra}$ assay results revealed that the positive signal was enhanced in the cell samples; the rate of signal enhancement reflects the level of metabolic activity. The data output is typically on a lifetime scale and can be transposed onto an $\mathrm{H}^{+}$scale, with acidification rates calculated by simple linear regression. Representative data showing the effect of salidroside on acidification in SGC-7901 and MKN-45 cells are presented in (Figure.3B). The pH-Xtra data (lifetime scale) from SGC-7901 and MKN-45 cells show that $80 \mu \mathrm{M}$ salidroside reduced extracellular acidification by SGC-7901 and MKN-45 cells.

\subsection{Salidroside inhibits glycolysis through the downregulation of}




\section{ENO1/PKM2/GLUT1 expression in gastric cancer cells.}

To confirm the inhibitory effect of salidroside on glycolysis, an in vitro assay was carried out utilizing $80 \mu \mathrm{M}$ salidroside to treat gastric cells. Salidroside significantly inhibited glycolysis in the extracellular matrix of the cells. ENO1, PKM2 and GLUT1 play an important role in glycolysis. Salidroside prominently decreased the expression levels of ENO1, PKM2 and GLUT1 compared with those in control cells, as determined by immunofluorescence staining (Figure.4A).

Western blot analysis confirmed that treatment with $80 \mu \mathrm{M}$ salidroside reduced the ENO1, PKM2 and GLUT1 protein expression levels in SGC-7901 and MKN-45 cells. These results suggest that salidroside may inhibit glycolysis through ENO1, PKM2 and GLUT1 in gastric cancer cells (an anti- $\beta$-actin antibody was utilized for normalization, Figure.5A). Densitometric analysis was used to quantify the band intensities. The ENO1, PKM2 and GLUT1 expression levels were markedly lower in SGC-7901 and MKN-45 cells treated with $80 \mu \mathrm{M}$ salidroside than in the corresponding control cells (Figure.5B,C).

In addition, the PKM2, ENO1 and GLUT1 mRNA expression levels were markedly lower in the salidroside-treated groups of both gastric cell lines than in the corresponding control groups (GAPDH mRNA was used for normalization); (Figure.5 D). The mRNA expression levels of ENO1 and PKM2, two glycolysis-related factors, were measured using qPCR. Salidroside decreased the ENO1, PKM2 and GLUT1 mRNA levels in SGC-7901 cells and MKN-45 cells compared to these levels in the corresponding control cells. Moreover, the ENO1, PKM2 and GLUT1 expression 
levels were significantly lower in the salidroside treated groups of SGC-7901 and MKN-45 gastric cancer cells.

\subsection{Effects of salidroside on gastric cancer cell proliferation in vivo.}

To evaluate the effect of salidroside on the tumorigenic ability of gastric cancer cells in the animal model. we chose a widely accepted subcutaneous xenograft tumour model using SGC-7901 and MKN-45 cells. Subcutaneous inoculation of SGC-7901 and MKN-45 human gastric cancer cells $\left(5 \times 10^{6}\right.$ cells $\left./ 0.15 \mathrm{~mL} / \mathrm{site}\right)$ led to the formation of tumors at each site. Salidroside was administered once daily from day 10 to day 17 after inoculation of SGC-7901 and MKN-45 human gastric cancer cells (Fig. 6A).The mice were sacrificed 7 days after the administration and the tumor volume and weight were measured. Observation showed that the tumor volume and weight of the treatment group were smaller than the control group. (Fig.6B,C).

Immunohistochemical analysis confirmed that treatment with salidroside reduced the ENO1, Hexokinase2 (HK2) and GLUT1 protein expression levels in SGC-7901 and MKN-45 xenograft tumour. These results suggest that salidroside may inhibit glycolysis through ENO1, HK2 and GLUT1 in gastric cancer cells (Figure.6D).

\section{Discussion}

Numerous studies have not only contended that the benefits of salidroside include a high medicinal value and anti-radiation, anti-virus, and immune-regulating activity ${ }^{11,}$ 12) but have also highlighted its anticancer, cellular immunity-promoting and antioxidant effects ${ }^{10,17,18)}$. Currently, salidroside's powerful apoptotic effect has been detected in lung cancer cells, and salidroside seems to exhibit an antiapoptotic effect 
in non-tumour cells and a cytotoxic effect in breast cancer cells ${ }^{19}{ }^{20)}$. Salidroside decreased the level of phospho-p38 in A549 cells and downregulated the activity of the ROS-phospho-p38 signalling pathway ${ }^{21)}$. Furthermore, salidroside was found to induce the apoptosis of gastric, liver and colorectal cancer cells ${ }^{22,23)}$. The main reasons for the decrease in cell activity include the regulation of apoptotic gene expression $^{24)}$ and the insufficient energy supply. Few studies have been conducted to investigate the effect of salidroside on energy metabolism in cancer cells. To explore the underlying anticancer mechanism of salidroside, we first hypothesized that salidroside has different effects on energy metabolism, especially glycolysis, in gastric cancer cells. Extracellular acidification rate measurements can directly reflect the glycolysis rate of cancer cells, enabling us to understand the effect of salidroside on the metabolic capacity of cancer cells. The extracellular acidification rate of gastric cancer cells treated with a certain concentration of salidroside decreased significantly, indicating that the intracellular glycolysis rate was decreased, thus decreasing the acidification rate. Salidroside can be shown to affect the glycolytic ability of cancer cells. In addition, salidroside decreased the glycolytic ability of gastric cancer cells at the initial stage but did not significantly inhibit cell activity. Therefore, we hypothesize that there is a sequential relationship between salidroside's effect on inhibiting the glycolytic ability of gastric cancer cells and its effect on the activity of gastric cancer cells. That is, gastric cancer cells are sensitive to salidroside's ability to inhibit glycolysis and reduce the energy supply and, thus, further inhibit cell activity. However, the duration for which the glycolytic function of gastric cancer cells is 
inhibited and when it will affect the decrease in cell activity, which needs further study in the future.

Considering previous studies ${ }^{25,26)}$, we focused on the effects of salidroside on the expression of the key proteins (ENO1 and PKM2) in the aerobic glycolysis pathway in cancer cells and GLUT1, the key protein mediating glucose uptake by gastric cancer cells. PKM2 can interact with many tumor-related genes to accelerate carcinogenesis, including HIF-1 $\alpha$, Oct-4, STAT3 and beta-catenin ${ }^{27)}$. ENO1 regulates the proliferation and metastasis of gastric cancer cells by regulating the AKT signaling pathway ${ }^{28)}$. In other studies results revealed that SIRT3 may be a downstream target for STAT3 and STAT3 could regulate the expression of SIRT3 at the transcript level, which further affected the expression of glycolysis-related proteins, such as GLUT1 ${ }^{29)}$. The effects of salidroside on the expression of these key rate-limiting glycolytic enzymes in cancer cells were investigated by qPCR, immunofluorescence and Western blotting. Salidroside significantly inhibited glycolysis by downregulating the expression of ENO1 and PKM2 in SGC-7901 and MKN-45 gastric cancer cells. Moreover, salidroside downregulated the expression of GLUT1 in SGC-7901 and MKN-45 gastric cancer cells, reducing glucose uptake by these cancer cells and decreasing the supply of glucose to these cancer cells from the source. Salidroside, on the one hand, reduces the glycolytic ability and, on the other hand, weakens the glucose uptake ability of cancer cells. Synergism results between these two effects of salidroside, which limits the productivity of cancer cells and makes them unable to meet the energy requirements of massive cell division and 
proliferation. In turn, the lack of energy supply decreases the activity of cancer cells, inhibiting their proliferation and migration ability.

In this study, salidroside inhibited the growth of SGC-7901 and MKN-45 human gastric cancer cells in a concentration-dependent manner. In addition, the results confirmed that salidroside could inhibit glycolysis in SGC-7901 and MKN-45 human gastric cancer cells. However, the effect of salidroside on other kinases in the glycolytic pathway of cancer cells awaits investigation. The combination of drug intervention and gene silencing has recently become a model to explore the effect of drugs on gene expression, which can be considered in the next step. Further study is necessary to determine whether we can identify a small-molecule compound inhibitor of ENO1 and PKM2 to combine with salidroside and observe the inhibitory effect on cancer cells or synthesize a small-molecule compound based on the molecular structure of salidroside that can effectively inhibit the aerobic glycolysis of cancer cells. Our results show that salidroside has the potential to inhibit the proliferation and invasion of cancer cells and increase apoptosis. However, whether salidroside can be combined with commonly used first-line anticancer chemotherapeutic drugs needs to be tested by animal and clinical trials.

\section{Acknowledgements}

The authors thank the Central laboratory of the First Hospital of Lanzhou University for assisting with the experiments. This study was supported by the Key Technologies Research and Development Program (CN) (2017YFC0908302). 


\section{Conflict of Interest}

The authors declare no conflict of interest.

\section{Supplementary Materials}

The online version of this article contains supplementary materials. 


\section{Reference}

1) Tahara $T$, Okubo $M$, Shibata $T$, Kawamura $T$, Sumi $K$, Ishizuka $T$, Nagasaka $M$, Nakagawa Y, Arisawa T, Ohmiya N, Hirata I. Association between common genetic variants in pre-microRNAs and prognosis of advanced gastric cancer treated with chemotherapy. Anticancer Res. 34(9):5199-5204 (2014).

2) Hainaut $P$, Plymoth A. Cancer as a metabolic disease. Curr Opin Oncol. 24(1):56-57 (2012).

3) Hanahan D, Weinberg RA. Hallmarks of cancer: the next generation. Cell. 144(5):646-674 (2011).

4) Vander Heiden MG, Cantley LC, Thompson CB. Understanding the Warburg effect: the metabolic requirements of cell proliferation. Science. 324(5930):1029-1033 (2009).

5) Young $C D$, Anderson SM. Sugar and fat - that's where it's at: metabolic changes in tumors. Breast Cancer Res. 10(1):202 (2008).

6) Shi Y, Liu S, Ahmad S, Gao Q. Targeting Key Transporters in Tumor Glycolysis as a Novel Anticancer Strategy. Curr Top Med Chem. 18(6):454-466 (2018).

7) Hitosugi T, Kang S, Vander Heiden MG, Chung TW, Elf S, Lythgoe K, Dong S, Lonial S, Wang X, Chen GZ, Xie J, Gu TL, Polakiewicz RD, Roesel JL, Boggon TJ, Khuri FR, Gilliland DG, Cantley LC, Kaufman J, Chen J. Tyrosine phosphorylation inhibits PKM2 to promote the Warburg effect and tumor growth. Sci Signal. 2(97):ra73 (2009).

8) Zhao Y, Liu H, Riker Al, Fodstad O, Ledoux SP, Wilson GL, Tan M. Emerging metabolic targets in cancer therapy. Front Biosci (Landmark Ed). 16:1844-1860 (2011).

9) Piast M, Kustrzeba-Wojcicka I, Matusiewicz M, Banas T. Molecular evolution of enolase. 
Acta Biochim Pol. 52(2):507-513 (2005).

10) Fan $X J$, Wang $Y$, Wang L, Zhu M. Salidroside induces apoptosis and autophagy in human colorectal cancer cells through inhibition of PI3K/Akt/mTOR pathway. Oncol Rep. 36(6):3559-3567 (2016).

11) Noreen EE, Buckley JG, Lewis SL, Brandauer J, Stuempfle KJ. The effects of an acute dose of Rhodiola rosea on endurance exercise performance. J Strength Cond Res. 27(3):839-847 (2013)

12) Doncheva ND, Mihaylova AS, Getova DP. Antinociceptive and anti-inflammatory effects of Rhodiola rosea L. extract in rats. Folia Med (Plovdiv). 55(3-4):70-75 (2013).

13) Zhong X, Lin R, Li Z, Mao J, Chen L. Effects of Salidroside on cobalt chloride-induced hypoxia damage and mTOR signaling repression in PC12 cells. Biol Pharm Bull. 37(7):1199-1206 (2014)

14) Liang CC, Park AY, Guan JL. In vitro scratch assay: a convenient and inexpensive method for analysis of cell migration in vitro. Nat Protoc. 2(2):329-333 (2007).

15) Livak KJ, Schmittgen TD. Analysis of relative gene expression data using real-time quantitative PCR and the 2(-Delta Delta C(T)) Method. Methods. 25(4):402-408 (2001).

16) Faustino-Rocha A, Oliveira PA, Pinho-Oliveira J, Teixeira-Guedes $C$, Soares-Maia R, da Costa RG, Colaco B, Pires MJ, Colaco J, Ferreira R, Ginja M. Estimation of rat mammary tumor volume using caliper and ultrasonography measurements. Lab Anim (NY). 42(6):217-224 (2013)

17) Ju L, Wen $X$, Wang $C$, Wei $Y$, Peng $Y$, Ding $Y$, Feng L, Shu L. Salidroside, A Natural Antioxidant, Improves beta-Cell Survival and Function via Activating AMPK Pathway. Front 
Pharmacol. 8:749 (2017).

18) Wojcik R, Siwicki AK, Skopinska-Rozewska E, Wasiutynski A, Sommer E, Furmanowa M. The effect of Chinese medicinal herb Rhodiola kirilowii extracts on cellular immunity in mice and rats. Pol J Vet Sci. 12(3):399-405 (2009).

19) Rosselli S, Maggio AM, Raccuglia RA, Morris-Natschke SL, Bastow KF, Lee KH, Bruno M. Acid rearrangment of epoxy-germacranolides and absolute configuration of 1beta,10alpha-epoxy-salonitenolide. Nat Prod Commun. 5(5):675-680 (2010).

20) Zhao G, Shi A, Fan Z, Du Y. Salidroside inhibits the growth of human breast cancer in vitro and in vivo. Oncol Rep. 33(5):2553-2560 (2015).

21) Wang J, Li JZ, Lu AX, Zhang KF, Li BJ. Anticancer effect of salidroside on A549 lung cancer cells through inhibition of oxidative stress and phospho-p38 expression. Oncol Lett. 7(4):1159-1164 (2014).

22) Shi X, Zhao W, Yang Y, Wu S, Lv B. Salidroside could enhance the cytotoxic effect of LOHP on colorectal cancer cells. Mol Med Rep. 17(1):51-58 (2018).

23) Feng J, Niu P, Chen K, Wu L, Liu T, Xu S, Li J, Li S, Wang W, Lu X, Yu Q, Liu N, Xu L, Wang F, Dai W, Xia Y, Fan X, Guo C. Salidroside mediates apoptosis and autophagy inhibition in concanavalin A-induced liver injury. Exp Ther Med. 15(6):4599-4614 (2018).

24) Bohm I, Schild H. Apoptosis: the complex scenario for a silent cell death. Mol Imaging Biol. $5(1): 2-14(2003)$.

25) Zhou X, Yao K, Zhang L, Zhang Y, Han Y, Liu HL, Liu XW, Su G, Yuan WZ, Wei XD, Guan QL, Zhu BD. Identification of Differentiation-Related Proteins in Gastric Adenocarcinoma Tissues by Proteomics. Technol Cancer Res Treat. 15(5):697-706 (2016). 
26) Qiao H, Wang YF, Yuan WZ, Zhu BD, Jiang L, Guan QL. Silencing of ENO1 by shRNA Inhibits the Proliferation of Gastric Cancer Cells. Technol Cancer Res Treat. $17: 1533033818784411(2018)$.

27) Hua Q, Mi B, Xu F, Wen J, Zhao L, Liu J, Huang G. Hypoxia-induced IncRNA-AC020978 promotes proliferation and glycolytic metabolism of non-small cell lung cancer by regulating PKM2/HIF-1alpha axis. Theranostics. 10(11):4762-4778 (2020).

28) Sun L, Lu T, Tian K, Zhou D, Yuan J, Wang X, Zhu Z, Wan D, Yao Y, Zhu X, He S. Alpha-enolase promotes gastric cancer cell proliferation and metastasis via regulating AKT signaling pathway. Eur J Pharmacol. 845:8-15 (2019).

29) Yang Y, Cao Y, Chen L, Liu F, Qi Z, Cheng X, Wang Z. Cryptotanshinone suppresses cell proliferation and glucose metabolism via STAT3/SIRT3 signaling pathway in ovarian cancer cells. Cancer Med. 7(9):4610-4618 (2018). 
Table 1. The information of primer in PCR assay

\begin{tabular}{llll}
\hline Gene & Accession number & Direction & Sequence \\
\hline \multirow{2}{*}{ ENO1 } & \multirow{2}{*}{ NM_001201483 } & Forward & 5'-GCGCATTGGAGCAGAGGTTTAC-3' \\
& & Reverse & 5'-CCATGCCGATGACCACCTTATC-3' \\
PKM2 & \multirow{2}{*}{ NM_001206799 } & Forward & 5'-CATGTCGAAGCCCCATAGTGA-3' \\
& & Reverse & 5'-AACGTCTCCACTGATCGGGAAG-3' \\
GLUT1 & \multirow{2}{*}{ AY034633.1 } & Forward & 5'-CATTGGCTCCGGTATCGTCAAC-3' \\
& & Reverse & 5'-ATGGCCACGATGCTCAGATAGG-3' \\
GAPDH & NM_002046 & Forward & 5'-CCTCTGACTTCAACAGCGACAC-3' \\
& & Reverse & 5'-GCCAAATTCGTTGTCATACCAG-3' \\
\hline
\end{tabular}



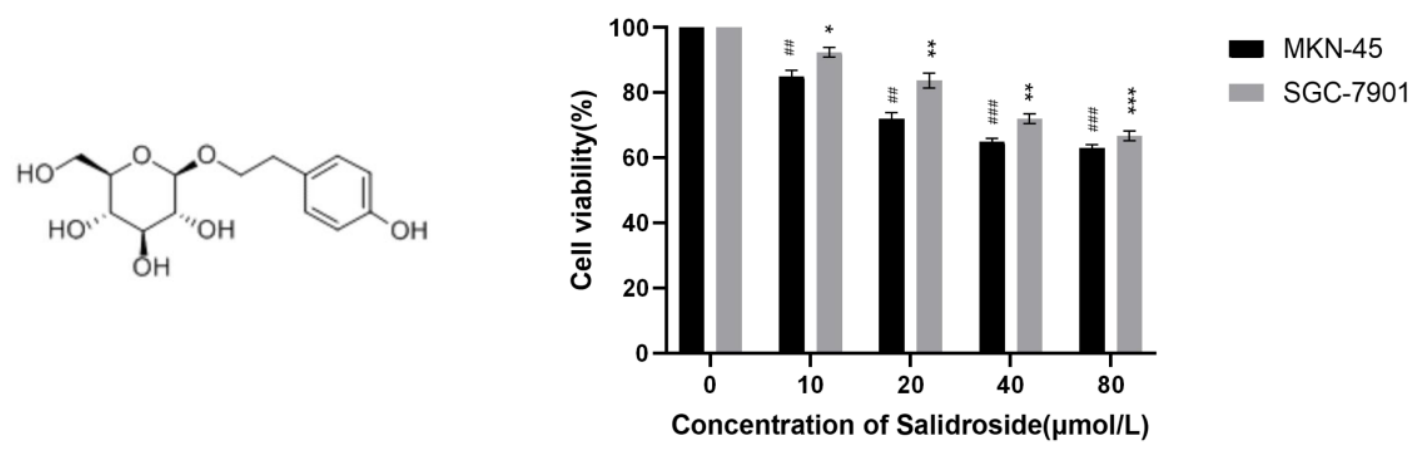

Figure. 1 The effect of salidroside on the activity of tumour cells.

(A) Schematic diagram showing the procedure for the experiment. (B) The SGC-7901 and MKN-45 cells were treated with various salidroside concentrations for $24 \mathrm{~h}$. The viability of cells was determined by CCK- 8 assay. $* / \#=p<0.05, * * / \# \#=p<0.01$ and ***/\#\#\#=p<0.001 vs. the control group (* for SGC-7901, \# for MKN-45). 



Figure.2 Salidroside inhibited the migration of SGC-7901 and MKN-45 gastric

cancer cells. (A)Salidroside inhibited the migration of SGC-7901 and MKN-45 cells.

(B) Cell migration rate was analysed. $* * / \# \#=p<0.01$ and $* * * / \# \# \#=p<0.001$ vs. the control group $(*$ for SGC-7901, \# for MKN-45). scale bar=100 $\mu \mathrm{m}$. 

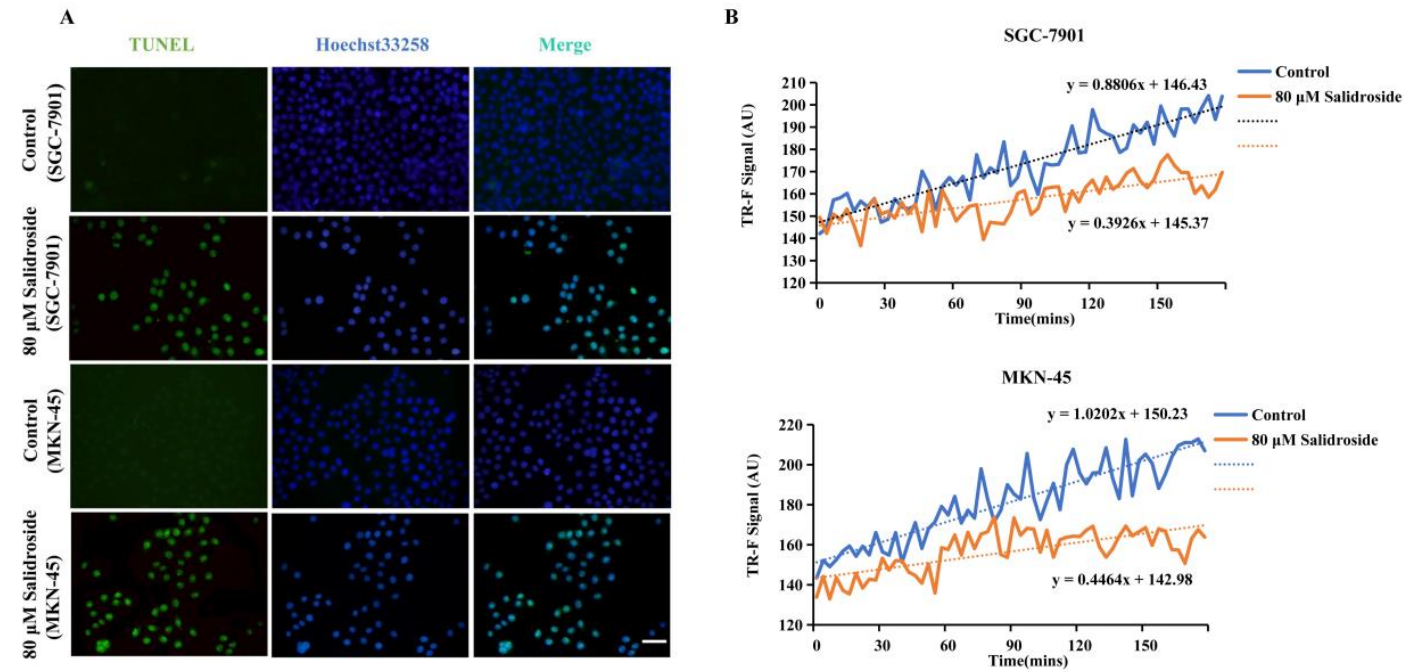

Figure.3 The ability of salidroside increase apoptosis in SGC-7901 and MKN-45

gastric cancer cells. (A) A TUNEL assay was adopted for detecting the morphological characteristics of apoptosis in SGC-7901 and MKN-45 cells for $24 \mathrm{~h}$ with $80 \mu \mathrm{M}$ salidroside treatment. The TUNEL experiment is a qualitative experiment rather than a quantitative, some representative visual fields were chosen which can't reflect the survival rate of cells. scale bar $=50 \mu \mathrm{m}$. (B) The ability of salidroside to inhibitory glycolysis in SGC-7901 and MKN-45 gastric cancer cells. SGC-7901 and MKN-45 cells were treated with $80 \mu \mathrm{M}$ salidroside exerted inhibitory effects on glycolysis by ECAR assay. (Color figure can be accessed in the online version.) 


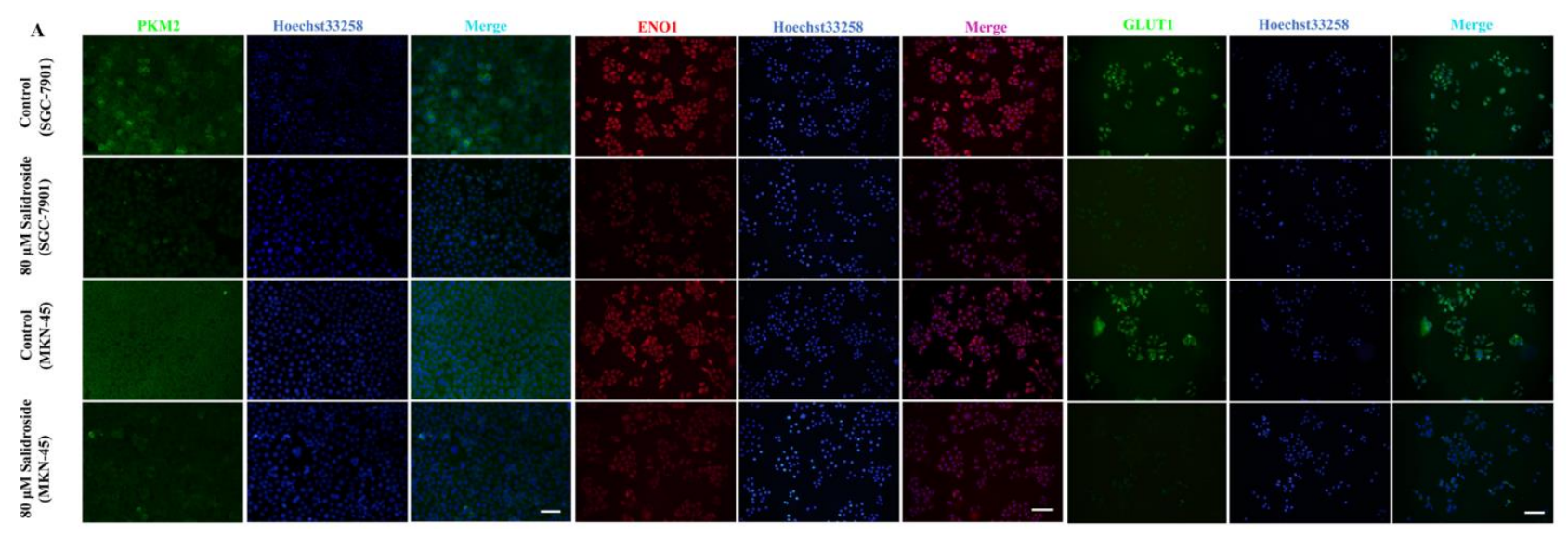

Figure.4 Salidroside inhibited glycolysis by downregulating PKM2, ENO1 and GLUT1 expression in SGC-7901 and MKN-45 gastric cancer cells. (A)Cells were treated with $80 \mu \mathrm{M}$ salidroside for $24 \mathrm{~h}$. Salidroside substantially decreased expression compared with that in the corresponding control cells, as determined by immunofluorescence staining. Scale bar $=100 \mu \mathrm{m}$. (Color figure can be accessed in the online version.) 

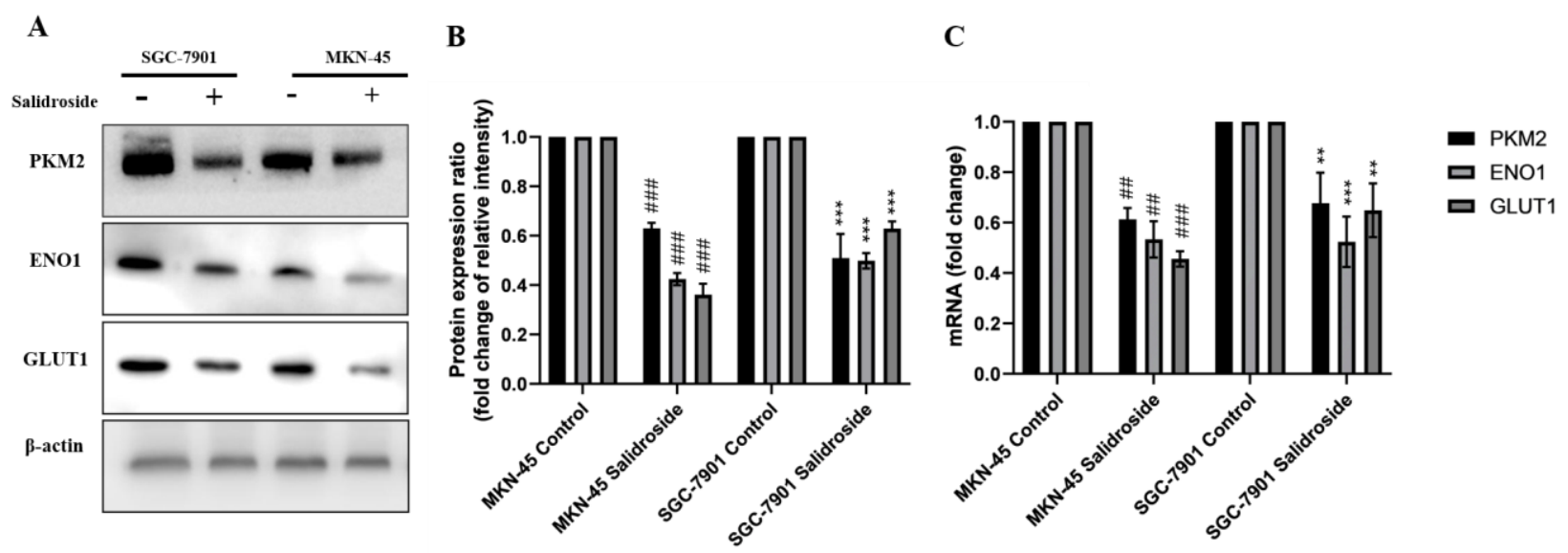

Figure 5. The expression levels of ENO1, PKM2 and GLUT1 (A) The protein expression levels of ENO1, PKM2 and GLUT1 were analysed using Western blot analysis. An anti- $\beta$-actin antibody was utilized for normalization. (B)The intensity of the bands was quantified using densitometric analysis. The ENO1, PKM2 and GLUT1 expression levels were significantly lower in $80 \mu \mathrm{M}$ salidroside-treated SGC-7901 and MKN-45 cells than in the corresponding control cells. (C) Effects of salidroside on the protein and mRNA expression levels of ENO1, PKM2 and GLUT1 in SGC-7901 and MKN-45 cells. The ENO1, PKM2 and GLUT1 expression levels in all treated cells were markedly lower than those in the corresponding control cells. GAPDH mRNA was used for normalization. All values are the means \pm SDs of three independent experiments. $* * / \# \#=p<0.01$ and $* * * / \# \# \#=p<0.001$ vs. the control group (* for SGC-7901, \# for MKN-45). 

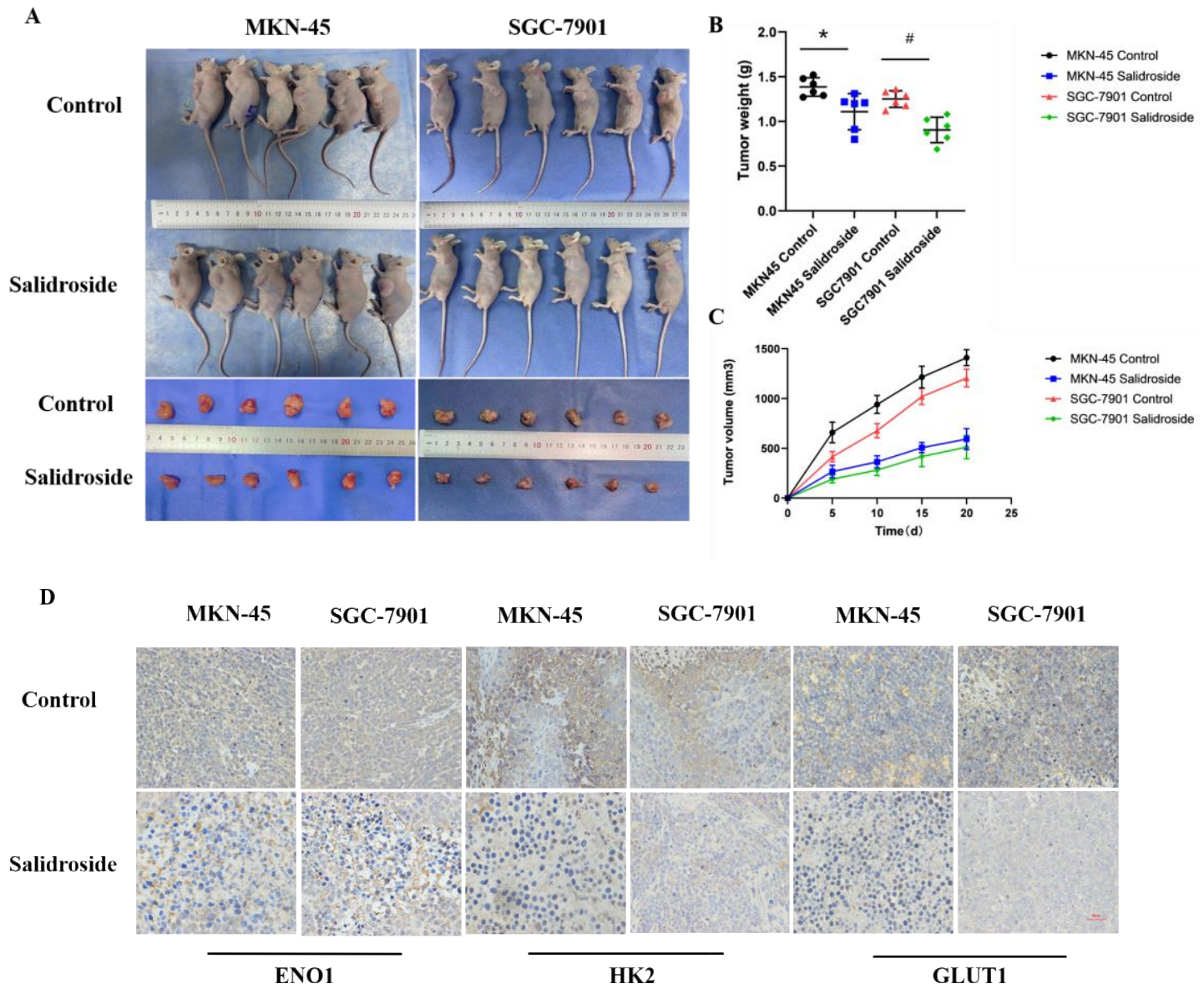

Figure. 6 Salidroside inhibited the growth, invasion and metastasis of gastric cancer cells in vivo. (A,B) After 17 days, tumors were excised after the final treatment. (C) The tumour volumes and weights were measured and the treated groups were markedly smaller than those in the corresponding control groups. (D) Effects of salidroside on the protein expression levels of ENO1, HK2 and GLUT1 in SGC-7901 and MKN-45 xenograft tumour. The ENO1, HK2 and GLUT1 expression levels in all treated cells were markedly lower than those in the corresponding control xenograft tumour. Scale $\operatorname{bar}=50 \mu \mathrm{m} . * / \#=p<0.05$ vs. the control group $(*$ for SGC-7901, \# for MKN-45). (Color figure can be accessed in the online version.) 